\title{
Biological tissue adhesive for multiple use in the accident and emergency department
}

\author{
Claire Gerrard, Steve Moore, Brendan Ryan
}

\begin{abstract}
Objective-To assess the strength of the glue and microbial contamination over 28 days from opening a vial of tissue adhesive in the accident and emergency setting, and to quantify cost savings of repeated use of the vials.

Method-(1) Strips of reinforced nylon and a specially constructed piece of apparatus designed to measure the force at which the glue gave way were used to measure the strength of the tissue adhesive at various times after the glue was opened to assess if the glue strength deteriorated over time. (2) Microbial contamination of the glue was assessed.

Results-There was no deterioration in the glue strength over time. There was no evidence of microbial contamination of the glue.

Conclusion-Cyanoacrylate tissue adhesive can safely be reused for a period of 28 days after opening with no risk of degradation of glue strength or contamination with micro-organisms. In our department this represents a potential saving of $£ 5400$ per year.

(F Accid Emerg Med 2000;17:341-343)
\end{abstract}

Keywords: biological tissue adhesive

Tissue adhesives have many advantages over other methods of minor wound closure including ease and speed of application, ${ }^{1}$ relatively painless application and dressings are not required. ${ }^{3}$ They are used frequently and successfully in many accident and emergency (A\&E) departments.

The tissue adhesive used in our department is Indermil. This is presented in a $5 \mathrm{~g}$ vial. The manufacturers recommend this for single use only. We estimated that the amount of glue used on a $2 \mathrm{~cm}$ wound was less than $0.05 \mathrm{~g}$ and hence the $5 \mathrm{~g}$ vial would suffice for up to 100 such wounds. We therefore sought to determine the feasibility of the repeated use of a vial of tissue adhesive in regard to its strength and contamination by micro-organisms.

Accident and Emergency Department, Wythenshawe Hospital, Southmoor Road, Manchester

Correspondence to: Dr Gerrard

(clairegerrard@hotmail.com)

Accepted 29 February 2000

\section{Methods}

The glue we tested was Indermil taken from a $5 \mathrm{~g}$ vial.

Initially a simple calibration test was performed to test the consistency of the apparatus, using 18 similar sized strips of A4 office quality paper. The strips of paper were placed into the testing apparatus in the same way as the nylon strips and then force was applied. The range of forces at which the paper ripped was 83.4 to
107.9 Newtons (mean 95.8; standard deviation 6.3). The coefficient of variation for the calibration experiment was $6.6 \%$, indicating that the test apparatus was consistent and reliable.

A pilot study was then carried out using 30 strips of reinforced nylon. The forces at which the glue gave way ranged from 11.6 to 86.0 Newtons (mean 46.2; standard deviation 20.2) with a coefficient of variation of $43.8 \%$. Comparison of this value with that obtained from the calibration experiment indicates that the variation in the performance of the glue was considerably greater than the variation in the performance of the apparatus.

\section{STRENGTH ASSESSMENT}

The model used to assess glue strength was reinforced nylon. Although this is not what the glue is designed for, it was suitable for our experiment because the material was such that it did not tear in the force gauge before the glue bond gave way - that is, material stronger than the glue had to be used. The actual material did not matter, as all we were testing was strength of the glue on subsequent occasions.

Two drops of the glue were applied to strips of the reinforced nylon and two of these strips were then glued together with a uniform amount of overlap and held together for 30 seconds using a $1 \mathrm{~kg}$ weight. The strips were then pulled apart using a purpose built electronic force gauge (fig 1). The force required to disrupt the bond was recorded. Three different tubes of glue were used. For each of the tubes 24 strips were glued together, at $1,7,14,21$ and 28 days after opening the tube. From each group six strips were tested at 1 hour, 24 hours, 48 hours and 72 hours after gluing had taken place.

TO ASSESS THE RISK OF CONTAMINATION OF THE GLUE AFTER REPEATED USE

One drop of glue was dropped onto six blood agar plates, six CLED plates (cysteine, lactose

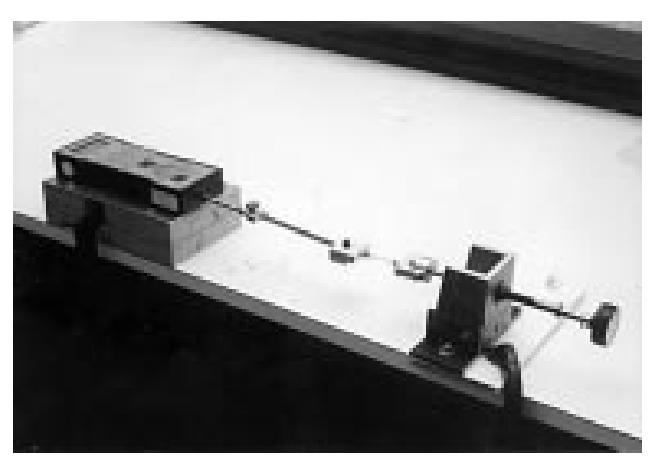

Figure 1 The purpose built electronic force gauge. 
Table 1 Means (SD) of original data on subsequent days after opening

\begin{tabular}{lll}
\hline & Mean (Newtons) & $S D$ \\
\hline Day 1 & 27.362 & 8.967 \\
Day 7 & 37.129 & 12.261 \\
Day 14 & 33.060 & 11.587 \\
Day 21 & 39.783 & 14.203 \\
Day 28 & 37.957 & 11.859 \\
\hline
\end{tabular}

electrolyte deficient) and into six bottles of nutrient broth and stored over night at $4^{\circ} \mathrm{C}$. The above procedure was repeated and the culture dishes were then incubated at $30^{\circ} \mathrm{C}$ and $37^{\circ} \mathrm{C}$.

This process was repeated at 7 and 14 days after opening the vial.

These culture plates and nutrient broths from day 1, 7 and 14 all remained sterile. To verify that this result was genuine and was not attributable to an unsuitable host medium, we then adopted a different approach.

A sample of glue from a new tube was added to different suspensions contaminated with Staphylococcus aureus, Pseudomonas aeruginosa, Escherichia coli and Enterococci. Controls were set up without the glue and all the suspensions were incubated over night at $37^{\circ} \mathrm{C}$.

This process was repeated on tubes at different stages throughout the experiment (days 1, 14 and 28 ).

The nozzles of the tubes being tested were swabbed at three different stages throughout the experiment (days 1, 14 and 28) to check for contamination of the tube. At the same time the tubes of glue that were being used repeatedly within the department (in the clinical setting) were swabbed at various stages throughout the research period.

\section{Results}

Figure 2 and table 1 summarise the results of the full study. No significant differences were found between the different tubes of glue tested on each day. However, the mean force required to achieve glue failure (separation) was significantly greater on each of days 7,14 , 21 and 28 than on day 1 .

The forces at which the paper strips ripped in the calibration study and at which the glue gave way (that is, strips separated) in the pilot and full experiments were found to follow normal distributions. All data were thus evaluated using parametric statistical methods and results are presented as means with their standard deviations. To avoid problems of multiple testing, comparisons between days

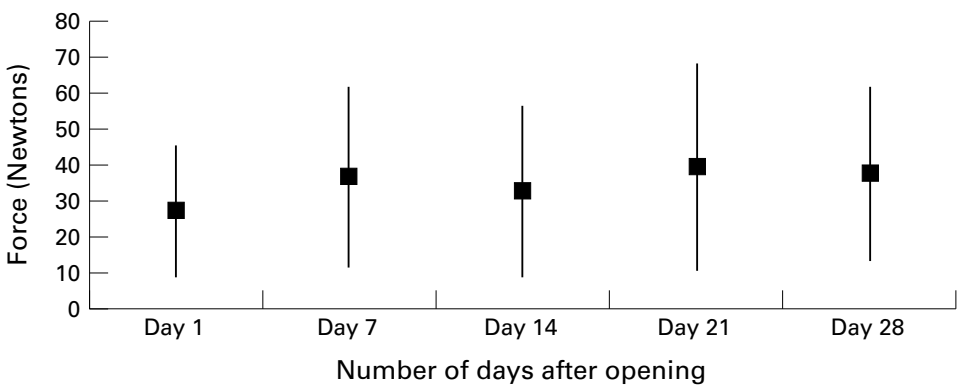

Figure 2 Graph to show the mean and range of strength tests. Mean (squares) and 2SD (lines) are shown. and between vials were carried out using analysis of variance methods. All calculations were done using the SPSS statistical software package in conjunction with the Medical Statistics Research Support Unit in the University of Manchester Medical School.

\section{STATISTICAL POWER CALCULATION}

The mean (SD) shearing force required to separate glued strips in the pilot study was approximately 45 Newtons. A one third reduction (thus $15 \mathrm{~N}$ ) in this force because of deterioration in the adhesive properties of the glue over time was considered to be clinically significant. To detect a difference of this magnitude would require 17 replicate observations per experiment. Each experiment actually consisted of six replicate observations on each of three vials (total 18 observations) with differences between vials ignored for the evaluation of the primary study objectives (evaluation of changes over time).

All the plates that had had the glue added to them showed no microbiological growth, whereas all the controls without glue had grown colonies of bacteria.

All swabs taken from the bottles were negative, and the glue was actually found to have a bacteriostatic effect on the bacteria tested. The swabs from the tubes being used in the clinical setting also remained sterile.

\section{Discussion}

This study shows that the strength of the glue tested does not deteriorate over a period of 28 days after opening.

The statistical analysis showed significant differences between the glues strength on the different days tested. These differences were however, in the opposite direction to that expected. Rather than the glue becoming "weaker", it actually became stronger and more adhesive with time. One possible reason for this is a deterioration in the glue making it "stickier" but also less pliable (a phenomenon that usually manifests itself in the plastic mass that accumulates around the nozzle of a tube of glue with increasing use). Potentially, if the glue becomes less pliable, it may also become less likely to give reliable results. This did not happen. Increased glue strength seems to be gained over 28 days without any apparent deterioration in glue pliability or usability.

We also feel that the small number of results that we received that were less than $15 \mathrm{~N}$ (one result as low as $6 \mathrm{~N}$ ) were the result of the influence we had over the way the strips were glued and also the way they were placed into the apparatus before the strength test took place. If the strips were not placed exactly straight into the grips, and a slight angulation or twist occurred, the glue bond, when force was applied was subject not only to a shearing force but also to a degree of peeling force, and therefore, the bond gave way sooner and the result that was recorded was biased. There were only a small number of occasions when this took place. These results were, however, included in the analysis. 


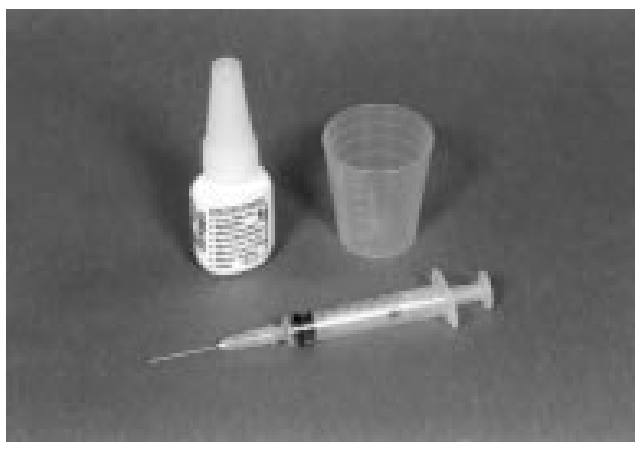

Figure 3 Medicine pot, needle and micropipette for dispensing the glue.

In clinical use glue strength is adequate at day 1 after opening. We have shown experimentally that this strength does not deteriorate over time.

The results received from the microbiology investigations showed no evidence of contamination of the glue when it was stored under normal clinical conditions. However, there is still a potential risk of viral transfer. In view of this we would suggest the following alternative ways of dispensing the glue. The glue could be dispensed from the tube via a 25 gauge sterile needle attached to the nozzle, replacing the needle for each application, or you could dispense the glue into a medicine pot and then apply it with a small syringe and needle or a micropipette (fig 3). Another possibility would be to dispense the glue into a venflon, detach it from the tube and then apply to the wound. This method would avoid all possible cross contamination.

We have calculated a cost saving of $£ 5400$ per year based on $5 \mathrm{~g}$ single vials of glue used for multiple applications in our department. We calculated an approximate use of once per day in our department, with each vial being used for up to 28 days. Therefore, $£ 16.50 \times$ $(365 / 28)=£ 215.09$ year $(£ 16.50=$ cost of one vial)

Compared with cost on a "once only" basis, $£ 16.50 \times 365=£ 6022.50$ year
The final cost saving of $£ 5400$ takes into account monies used for application materials (venflons, sterile needles, etc).

Many A\&E departments already use cyanoacrylate tissue adhesive repeatedly. Our evidence supports its use in this way. However, legal implications must be taken into account if manufacturers' recommendations are disregarded, and it must be remembered that our experiment was conducted "in vitro" and not in the clinical setting. Further investigation may therefore, be warranted.

\section{Conclusions}

(1) The adhesive properties of this tissue adhesive do not deteriorate after the vial has been opened for a period of up to 28 days.

(2) There was no evidence of contamination of the opened vials when they were stored in the standard clinical setting.

(3) It would be possible to use tissue adhesive on a repeated basis if a reliable delivery system can be devised.

(4) Considerable cost savings to $\mathrm{A} \& \mathrm{E}$ departments would arise if repeated use of tissue adhesive was to be implemented.

\section{Contributors}

Claire Gerrard designed the original study and method and was responsible for data collection, analysis, interpretation of results and writing of the paper. Steve Moore participated in design of the paper, discussed issues involved with practical aspects and edited the paper. Brendan Ryan coordinated the research, supervised the study and edited the paper. Brendan Ryan is the guarantor of the paper. Kevin Reynard edited the paper. Dave Weston and Angela Downs (Department of Microbiology, University Hospital of South Manchester) participated in the practical aspects and advised on the microbiological testing of the glue. Mike Bolton and John Meade (Clinical Engineering, University Hospital of South Manchester) designed the force gauge apparatus. Brian Faraghar (Medical Statistics Research Support Unit, University of Manchester) advised on statistical methods and significance.

Funding: none.

Conflicts of interst: none.

1 Maw J, Quinn J. Cyanoacrylate tissue adhesives. Am $\mathcal{f}$ Cosmetic Surg 1997;14:413-16.

2 Watson DP. Use of cyanoacrylate tissue adhesive for closing facial lacerations in children. BMF 1989;299:1014.

3 Noordzij JP, Foresman PA, Rodeheaver GT, et al. Tissue adhesive wound repair revisited. F Emerg Med 1994;12: 645-9. 